\title{
Characterization of Classification, Function and Enzymolysis Mechanism of Cellulase
}

\author{
Di Chen ${ }^{1}$, Qianqian Tang ${ }^{1}$, Yuxian You ${ }^{1}$, Yiwen $\mathrm{Li}^{1}$, Siqi Zeng ${ }^{1}$, Chaohui Feng ${ }^{1}$, \\ Hejun $\mathrm{Wu}^{1}$, Aiping $\mathrm{Liu}^{1}$, Cheng $\mathrm{Li}^{1}$, Yuntao $\mathrm{Liu}^{1,2, \mathrm{a}^{*}}$ \\ ${ }^{1}$ College of Food Science, Sichuan Agricultural University, Yaan 625014, China. \\ ${ }^{2}$ Animal Nutrition Institute, Sichuan Agricultural University, Chengdu 611130, China. \\ a*lyt_taotao@163.com (corresponding author)
}

Keywords: Cellulase, Enzyme activity, Molecular structure, Function mechanism, Molecular biology

\begin{abstract}
As the richest polysaccharide resource in the nature, cellulose has a great development and utilization value. An in-depth research into cellulase is the prerequisite linchpin to the development and utilization of cellulose. Therefore, this paper conducts a comprehensive summary and analysis of the classification, the measurement method of determination of enzyme activity, the molecular structure and functions, the enzymolysis mechanism and the molecular biological study of cellulase, aiming at showing the current research status of the cellulase.
\end{abstract}

\section{Introduction}

Cellulose is the most widely-existing sugar with the highest content. It has an extensive range of applications in the modern industry, such as paper-making, spinning, feed and food additives. The biological degradation phenomenon of cellulose was discovered early in 1886. The cellulase is a complex enzyme system. Research methods into cellulase have undergone three stages, including biochemical methods, genetic engineering and structural biological methods, methods protein engineering methods. [1] With the development of research methods, research findings related to cellulase have been kept on updating.

\section{Overview of Cellulose}

Cellulose is the richest organic matter in the biosphere [2] and is supplemented through the photosynthesis. [3] Cellulose is the polysaccharide featuring the structure of plants (including some fungi and bacteria) and is the major constituent of plants' cell wall, accounting for more than $50 \%$ of carbon of the plant kingdom. The plant cellulose generated through the photosynthesis all over the world is about 200 billion tons annually, of which $89 \%$ has not yet been utilized by humans, and about $11 \%$ is used as forage grasses, papermaking and architectural raw materials [4].

\section{Classification of Cellulase}

The complexity of the structure of cellulose leads to the difficulty of efficiently dissolving it through any single enzyme. The cellulase which can hydrolyze the natural cellulose is a complex multi-enzyme system. The cellulase system is made up of three enzymes with different functions but completing each other. There are three types of enzymes. The first type is endoglucanase or endo-1,4- $\beta$-D-glucanohydrolase, which is abbreviated as EG or BGL. Since carboxymethylcellulose (CMC) is employed as the substrate to test its vitality, it is also called CMC enzyme. EG can randomly hydrolyze non-crystalized cellulose including the CMC inflated by phosphoric acid, the inflated cellulose, the cellodextrin generated during the degradation of fibers, and release cello-oligosaccaride to form new reduction ends. The degradation capability of cellulose weakens with the increase of the restoration end and the bond length; The exoglucanase includes two kinds of enzymes, including 
cellodextrin and exo- $\beta$-1,4-glucanase or cellulose disaccharide hydrolase, which is shortened as cellobiohydrolases or $\mathrm{CBH}$. $\mathrm{CBH}$ can hydrolyze fibers with a high crystallinity, including microscrystalline cellulose and cotton. The choice of $\mathrm{CBH}$ about the direction of cellulose chain is targeted while hydrolyzing cellulose. Some hydrolyzes $\beta-1,4$-glucoside from the non-reducing sugar end to generate the cellobiose. Some releases the cellobiose from the reducing end. A minority of $\mathrm{CBH}$ excises the single glucose residue to generate glucose; $\beta$-1,4-glucosaccharase which is shortened as $\beta$-glucosidase (BGL) or cellobiase (CB). BGL is mainly used to hydrolyze CB and cello-oligosaccharide to generate glucose. Strictly speaking, BGL cannot be counted as cellulase, but it can alleviate the feedback and inhibiting function of CB against the cellulase. [5-7]

\section{Methods to Measure the Cellulase Activity}

Since the 1960s, many scholars have studied the measurement methods of the cellulase activity. Cellulase has some characteristics rare to find among the other enzymes. Among numerous researchers put forward different measurement methods, Ghose has been widely recognized [8]. In 1984, the Fermentation Committee of the International Union of Pure and Applied Chemistry (IUPAC) confirmed that the enzyme activity measurement based on the filter paper has been defined as a standard method. According to research findings of the lower fungus cellulase, such as Trichoderma, the measurement methods of the cellulase once published are summarized, including cotton cutting method, filter paper collapse method, nephelometry, measurement method featuring the reduction of viscosity and generation of reducing power with CMC-Na as substrate, Barnch and swain measurement method, dyeing cellulose method, rapid measurement method for CMC liquidated activity of the cellulase and flat band method. The generation, secretion and function mechanism of the enzyme of some microorganism whose fibrinolysis capability of bacteria is strong are seldom studied, and the above stated measurement methods are not applicable [9].

\section{Molecular Structure and Function of Cellulase}

Though physicochemical property and catalytic activity of cellulase of different sources are not totally the same, the size of the cellulase molecules features a wide range. The molecular weight of the general endo-enzyme is within the scope of 23 146kDa. For example, EG of the fungi is made up of two isomerases, including EG I and EG III, the molecular weight of EG I is about 54kDa and that of EG III is about $49.8 \mathrm{kDa}$. The molecular weight of the exo-enzyme is about $38 \sim 118 \mathrm{kDa}$. For example, $\mathrm{CBH}$ of Trichoderma has two isomerases, namely CBH I and CBH II. The molecular weight of CBH I is about $66 \mathrm{kDa}$; while that of $\mathrm{CBH}$ II is about $53 \mathrm{kDa}$. The molecular weight of $\beta$-glucanase is 90 100kDa (endoenzyme) and 47 76kDa (extracellular enzyme) [10].

The cellulase of most fungi and a few bacteria will suffer glycosylation. The glycosyl and the protein combine with each other in the form of covalent bond or in the dissociable complexing state. The glycosylation protects enzyme from suffering the hydrolysis of protese to some extent. Due to glycosylation, celluse differs its ratio of carbohydrate between different enzymes, thus resulting in multiple forms of enzymes and differences of molecular weights.

After studying the primary structure and the tertiary structure of the cellulase, researchers found out that molecules of cellulase share a similar structure, which is made up of three parts, namely linker, cellulose-binding domains (CBD) and spherical catalytic domains (CD).

1) Linker: Most of the linker area of cellulase is rich in proline (pro), hydroxyl-amino-acid (Ser, Thr) and Arg, Glu and Gln. The role of linker is to keep the distance between CD and CBD, and can help different enzyme molecules to form a relatively stable aggregation.

2) CBD: CBD is not necessary for catalytic activity of enzymes. However, it gives full play to the special activity of regulatory enzyme on soluble and insoluble substrates. The function mechanism of the combined cellulose has not yet been clear. Some even thought that CBD stabilizes the crystalized substrates through the stabilization of hydrogen bonds. Most CBD of cellulose is located in C-end or $\mathrm{N}$-end of protein. Seldom is it located in the middle. 
3) CD: It shows the catalytic activity of enzymes and their characteristics of interacting with specific water soluble substrates. Though molecular weights of cellulase from different resources differ greatly, the size of their CD is almost the same. [11] Currently, the most in-depth research into the tertiary structure of cellulase is about CBH I and CBH II of T. reesei. The dewatering cluster analysis method and the small-angle X-ray diffraction analysis are employed to find out that: both CBH I and CBH II molecules both feature a tadpole shape and a length of about 10nm. The "tail" made up of about 30 amino acid residues is the CBD of cellulose. The core protein (CP) in the "head" features a round shape and contains the catalytic center. The "tail" and "head" are connected by a soft linker of about $5 \mathrm{~nm}$ long.

\section{Function Mechanism of Cellulase}

Function mechanism has been an issue at the core of research into cellulase. Over the past few years when Reese and other scholars put forward C1-Cx hypothesis in the 1950s for the first time to the emergence of molecular biology of the modern cellulose, scholars in various countries have made various researches. With the development of the biological technique, the exposition of enzymolysis mechanism from the perspective of the molecular level has been a possibility. The cellulase molecule is generally made up of linker, CBD and CD. However, there have not yet been sensible explanations as to how CBD was absorbed to the surface of cellulose and how CBD interacts with CD to degrade cellulose [12]. Below are hypotheses having been put forward up to now.

C1-Cx Theory. The theory means that C1 enzyme first functions on the crystalline cellulose to make it become the non-crystalized cellulose. Cx randomly hydrolyzes the non-crystalized cellulose to generate soluble cellulose and $\beta$-1,4-oligomer of glucose. Then, $\beta$-glucanase hydrolyzes CB and cellotriose into glucose.

Sequence Function. This function means that the endoglucanase first functions on $\beta-1,4$ glucosdic bond of cellulose to open a gap. Then, CB hydrolase of the exoglucanase cuts a dimer at the no-reducing end. $\beta-1,4$ glucanase hydrolizes CB into glucose.

Synergistic Function. The hydrolysis of cellulose calls for the synergy of three ellulases. Some reports pointed out that the synergistic effect of exoglucanase and endoglucanase with more compact natural cellulose is more obvious. There is with a synergistic function among the full-value cellulase system which can degrade the crystallized and non-crystalized substrates. It should be noted that the function sequence of enzymes is not absolute and that the functions of various enzymes are not simple and fixed. Besides, the research findings of Wood [13] about the function style of different enzyme groups generated by penicillium pinophilum have supported the idea about the continuous synergistic function style between CBH and EG in cellulose solution. Results suggest that, unless different CBHs and two different EGs are put together to cause some function, hydrolysis is nonsense. Generally speaking, the synergistic function is in direct proportion to the crystallinity of enzymolysis substrates. When the mix proportion of enzyme components is closer to the percentage of various enzyme groups in the bacteria fermentation broth, the stronger the synergistic function is. There is also a synergistic function between exoglucanase and endoglucanase from different bacteria [14, 15]. The multi-enzymes of cellulase is a must to the launch of mass hydrolysis. A series of researches have suggested the synergistic effect of various enzymes in the biological cellulase system is relatively complex. There are five expression forms in total: 1 ) The synergistic function between endoglucanase and $\mathrm{CBH}$; 2) The synergistic function between different CBHs; 3) The synergistic function between different endoglucanases; 4) The synergistic effect between molecules; 5) The synergistic effect between exoglucanase and $\beta$-glucossacharase [16].

Disordering Degradation Mechanism. Some scholars thought that, before synergistic degradation of cellulose by the cellulase, the cellulose crystallization zone turns into the disordering one $[17,18]$ and short fibres are formed [19]. The disordering response before synergistic degradation by enzymes is completed by a protein called C1. The hydrogen bond formed by it and the cellulose is stronger than the hydrogen bond between fibers. 
Oxidability Degradation Mechanism. The oxidability degradation mechanism was discovered while studying the wood degradation by brown-rot-fungi. First, though brown-rot-fungi can degrade a large number of woods, it can just lead to the significant decrease of the cellulose polymerization degree without causing weight loss. Then, though brown-rot-fungi is efficient in degrading cellulose, its cellulase system is not complete [20]. It does not have the exoglucanase, which is essential to hydrolysis of the crystalline region. Besides, during the wood degradation by brown-rot-fungi, the S2 layer of the plant cell wall is first degraded; while the S3 layer closely clingining to the hypha is preserved complete [21]. It can be predicted there is a unique and non-enzymatic wood degradation system. There might be a small molecule in the system which can enter the S2 layer of the cell wall, thus resulting in the depolymerization of cellulose [22]. Conclusions by a large number of researches show that: brown-rot-fungi might degrade cellulose in a non-enzyme style, with the participation of small molecules and involving of the oxidation mechanism.

\section{Molecular Biological Research into Cellulase}

Biological research into cellulase started from the gene cloning of cellulase in the late 1970s and is rapidly developed over the recent three decades. Up to now, there have been more than 400 cellulase genes having been cloned, expressed and sequenced. Trichoderma reesei alone has more than 30 genes due to its coming from different regions and having different bacterial strains, including genes of endoglucanase (EG I and EG III) and CB of exoglucanase (CBH I and CBH II) have been cloned and sequenced, and expressed in the Escherichia coli or saccharomycetes. Due to peoples' demands for cellulase, especially demands of industrial production, academic research focus is on the high-yield fungi and thermoduric bacteria of cellulase. Almost all cellulase genes which have been cloned especially those relate to endoglucanase and $\beta$-glucosaccharase have been expressed in Escherichia coli. However, the expression rate of endoglucanase and $\beta$-glucosaccharase is low and most enzymes generated cannot be secreted outside cells. The cloned cellulase genes mostly use the original promoters on the clone fragments. In order to obtain the exocytosis products, some cellulase gene projects has been efficiently expressed in bacillus subtilis, bacillus stearothermophilus, brevibacterium lactofermentum, streptomyces lividans, etc [23]. However, since the expression of the cellulase gene in the heterologic host will encounter problems like protease hydrolysis and glycosylation, and the secretion mechanism has not yet been clear, it is still a long way to go as to the large-scale industrial application. The expression of the cellulase genes in yeast is an important approach for the application development of the cellulose gene projects. However, the expression level of the exogenous cellulase genes by yeast is still limited. Therefore, the improvement of the cellulase's yield expressed by yeast will be an important research direction. The genetic modification of rumen microbes has been an important direction for molecular biology study. DNA engineering technique can be employed to increase the cellulose conversion rate in the cud of chewers' rumen, and significantly reduce the feed amount. At the same time, gene cloning can be employed to build efficient fibrinolysis engineering bacteria. The development of gene cloning technique has provided new methods for adjustment of cellulase synthesis, the degradation mechanism of cellulase and construction of new enzyme molecules.

\section{Acknowledgements}

This work was supported by Scientific Research Foundation of Sichuan Agricultural University (No. 06021400) and the National Natural Science Foundation of China (No. 31501550).

\section{References}

[1] B.X. Yan, F. Qi and Y.S. Zhang, Progress in structure-function studies of cellulases, Progress in Biochemistry and Biophysics. 26 (1999) 233-237. (In Chinese) 
[2] J.Y. Wang, S.G. Zhu and C.F. Xu, Biological chemistry, Higher Education Press, Beijing, 2002. (In Chinese)

[3] M.G. Adsul, K.B. Bastawde and A.J. Varma, Strain improvement of Penicillium janthinellum NCIM 1171 for increased cellulase production, Bioresource Technology. 98 (2007) 1467-1473.

[4] H.Z. Chen. Cellulose biotechnology, Chemical Indutry Press, Beijing, 2005. (In Chinese)

[5] D.D.Y. Ryu and M. Mandels, Cellulases: Biosynthesis and applications, Enzyme and Microbial Technology. 2 (1980) 91-102.

[6] H.V. Tilbergh, P. Tomme and M. CIaeyssens, Limited proteolysis of the cellobiohydrolaseI from T.reesei, MedSci entry for febs letters. 204 (1986) 223-227.

[7] Teeri, Crystalline cellulose degradation: New insight into the function of cellobiohydrolases, Trends in Biotechnology. 15 (1997) 160-167.

[8] T.K. Ghose, Measurements of cellulase activities, Pure and Applied Chemistry. 59 (1987) 257-268.

[9] L. Fu, Y.F. Ding and C. Zhang, Studies on methods for determination of cellulase activity, Journal of Xinjiang Agricultural University. 23 (2000) 45-48. (In Chinese)

[10] X.R. Wu, Cellulase enzyme molecular biology research progress and trends, Progress of Biotechnologhy. 14 (1994) 25-27. (In Chinese)

[11] P.J. Gao and Y.B. Qu, Advances of molecular biology in microbial degradation of cellulose, Journal of Cellulose Science and Technology. 3 (1995) 11-19. (In Chinese)

[12] B.X. Yan, Y.Q. Sun and P.J. Gao, Analysis of donmain's structure and function of cellulasese from Trichoderma Pseudokoningii S-38 by limited proteolysis, Journal of Cellulose Science and Technology. 6 (1998) 1-9. (In Chinese)

[13] T.M. Wood, S.I. Mccrae and S.I. Wilson, Aerobic and anaerobic fungal cellulases, with special reference to their mode of attack on crystalline cellulose Proc FEMS Symp Biochem Genet, Cellulose of Degradation. 31 (1988).

[14] T.M. Wood, Properties and models of action of cellulase, Biotechnology and Bioengineering Symposium. 5 (1975) 111-137.

[15] A.P. Moloney, S.I. Mccrae and T.M. Wood, Isolation and characterization of the 1,4- $\beta$-D-Glucan Hydrolysis of Talaromyces emersonii, Biochemical Journal. 225 (1985) 365-372.

[16] P.J. Gao and P. Xu, Resources and environment microorganism technology, Chemical Industry Press, Beijing, 2004. (In Chinese)

[17] M.P. Coughlan, The properties of fungal and bacterial cellulases with comment on their production and application, Biotechnology and Genetic Engineering Reviews. 3 (1985) 39-109.

[18] J.W. Koenings, Production of extracellular hydrogen peroxide and peroxidase by Wood-Rotting Fungi, Phytopathology. 62 (1972) 100-110.

[19] G. Halliwell, Hydrolysis of fibrous cotton and reprecipated cellulose by cellulolytic enzyme from soil microorganisms, Biochemical Journal. 95 (1965) 270-281.

[20] T.L. Highley, Cellulolytic activity of Brown-Rot and White-Rot Fungal on solid media, Holzforschung. 42 (1988) 211-216.

[21] T.L. Highley, L.L. Murmanis and J.G. Palmer, Micromorphology of degradation in western hemlock and sweetgum by the Brown-Rot Fungus Poria placenta, Holzforschung. 39 (1985) 73-78. [22] W. Wang and P.J. Gao, Research progress of lignocellulose degradation mechanism in Brown-Rot Fungus, Microbiology China. 29 (2002) 90-93. (In Chinese)

[23] F. Lin, Biochemistry and molecular biology research progress of cellulose, Life Sciences. 6 (1994) 18-22. (In Chinese) 